\title{
A NOTE ON THE ASSOCIATIVE LAW IN LOGICAL ALGEBRAS*
}

\author{
BY H. B. CURRY
}

1. Introduction. In 1925 Bernays published $\dagger$ a proof that within the formalism of the Principia Mathematica the proposition "Assoc" could be derived from the other primitive propositions. The purpose of this note is to give an alternative proof which brings out the fact that a similar conclusion holds in a variety of other systems, described hereunder as systems $B . \ddagger$ The proof is essentially a refinement of that given by Schröder in his Vorlesungen über die Algebra der Logik, vol. 1, pp. 255-257, and credited to C. S. Peirce. $\$$

2. Definition of Systems $A$ and $B$. A system $A$ is a system consisting of a class $K$ containing a rule of combination (which we may call multiplication and denote by simple juxtaposition), and a relation $<$ such that $\|$

I. $p<p p$.

II. $p q<q$.

III. $p q<q p$.

IV. If $p<q$ and $q<r$, then $p<r$.

A system $B$ is a system $A$ which has the additional properties:

$\mathrm{V}$. If $p<q$, then $r p<r q$.

VI. If $p<q$ and $p<r$, then $p<q r$.

THEOREM 1. If a system $A$ has either of the properties V, VI, it has the other, and so is a system $B$.

* Presented to the Society, January 2, 1936.

$\dagger$ Mathematische Zeitschrift, vol. 25, p. 312.

$\ddagger$ Bernays' proof is also valid in a system $B$. Indeed, he recognizes (footnote 6 , loc. cit.) that his proof is valid in more general systems, although he does not formulate any explicit limitations on them.

$\S$ Thus the present proof contains nothing essentially new and is probably known to several writers. The author has been led to publish it solely by the fact that the validity of Peirce's proof under these circumstances appears not to be universally realized; certainly it was overlooked by the authors of the Principia.

|| It should be recognized that I-VI are non-formal statements about the system concerned and not mere formulas. It is, of course, a weaker hypothesis that such rules hold than that formulas to the same effect are provable. 
Proof. (1) Suppose V holds. Then if $p<r$, we have $q p<q r$ by $\mathrm{V}$, and $p q<q p$ by III, hence $p q<q r$ by IV. If $p<q$, then $p p<p q$ by $\mathrm{V}, p<p p$ by I, hence $p<p q$ by IV. From these two conclusions we have $p<q r$ by IV, so that VI holds.

(2) Suppose VI holds. Then, if $p<q, r p<q$ by II, IV, and $r p<r$ by III, II, IV; hence $r p<r q$ by VI, and so V holds.

THEOREM 2. In any system $B$, if $p<q_{1}, p<q_{2}, \cdots, p<q_{n}$, and if $r$ is any combination of $q_{1}, q_{2}, \cdots, q_{n}$, then $p<r$.

Proof. The combination $r$ is constructed out of the $q_{i}$ by performing a number of times the operation of finding the product of two factors. At each stage of this process if the relation holds between $p$ and both of the factors, it holds between $p$ and the product by VI; since it holds between $p$ and all the $q_{i}$ it holds, by induction, between $p$ and $r$.

The property proved in Theorem 2 contains all forms of the associative law as special cases. For if $p$ is any combination which involves $q_{i}$, then $p<q_{i}$ by repeated applications of II, III, IV; as, for example, $q_{2}\left(q_{1} q_{3}\right)<q_{1} q_{3}<q_{3} q_{1}<q_{1}$. Hence we have the following theorem.

THEOREM 3. In any system $B$ the associative laws

$$
p(q r)<(p q) r, \quad(p q) r<p(q r), \quad p(q r)<q(p r)
$$

all hold.

3. Application to the Principia Mathematica. Here interpret $p q$ as $p \vee q, p<q$ as $\vdash . q \supset p$. Then I, II, III are Taut, $A d d$, and Perm, respectively, while IV and V both follow from Sum and the rule of inference. Hence $I-V$ all hold and the system is a system $B$ by Theorem 1 .

4. On the Converse of Theorem 3. That the associative laws may hold in a system $A$ in which $\mathrm{V}$ does not hold is shown by Bernays' second independence example. ${ }^{*}$ For here we may let $p q$ be interpreted as $p \vee q$ and let $p<q$ hold when and only when $\sim q \vee p=\alpha$. Then I-IV and the associative laws hold, while V fails for $p=\beta, q=\delta, r=\gamma$.

The Pennsyluania State College

* Loc. cit., Gruppe II on p. 318. 